\title{
S33. Psychosomatic disorders
}

THE CONCEPT OF PSYCHOSOMATIC DISORDER

\section{GA Fava}

Department of Psychology, University of Bologna, viale Berti Pichat 5, I-40127 Bologna, Italy.

Psychosomatic investigators have shown very little interest in a nosologic approach to their field. The concept of psychosomatic disorder is at present either poorly defined or strongly criticized, since it tends to perpetuate the obsolete notion of psychogenesis. In this latter instance, however, no alternative clinical conceptualization is proposed and reference to the multifactorial biopsychosocial model does not entail solution to the assessment of psychosocial problems of medical practice. The DSM-IV and ICD-10 diagnostic categories related to psychosomatic medicine offer very little help to the psychiatrist working in medical and surgical settings. They do not incorporate much research evidence which has accumulated in psychosomatic medicine in the past two decades. Such evidence involves the concept of abnormal illness behavior, studies concerned with quality of life, life events research and assessment of subclinical and prodromal symptoms of affective disorders. The clinical and heuristic value of the concept of psychosomatic disorder is then underscored, particularly in the presence of physical symptoms not explained by organic disease. There appears to be potential for psychosomatic specif1city, not in the form of disease specificity (certain illnesses viewed as psychosomatic in contrast with other diseases devoid of psychogenic etiology), but in the form of specific patterns of interaction of somatic and psychosocial symptoms.
Psychosomatic disorders, descriptive diagnosis and comorbidity

Harald J. Freyberger, H. Dilling

Department of Psychiatry (Director: Prof. Dr. H. Dilling), Medical University, Ratzeburger Allee 160, 23538 Lübeck, FRG

one the most important innovations distinguishing ICD-10, Chapter $V$ from traditional classificatory approaches concern the introduction of operational diagnosis and the concept of co-morbidity. Using psychopathological, time- and courserelated diagnostic criteria the concept of neurotic, psychosomatic and personality disorders have been changed following some des-oriptive principles. Based on data of multicentric studies in Germany for assessing acceptability, usefullness, goodness of fit and reliability of the new diagnostic categories dealing with these disorders, advatages and disadvantages of the diagnostic approach of ICD-10 were discussed. Ratings of psychiatrists and psychosomatic clinicians were contrasted with regard to the reception of the new diagnostic approach, especially concerning the principle of comorbidity.

The results were discussed with reference to multiaxial diagnostic approaches evaluating psychodynamic issues, which were excluded in descriptive diagnostic models. 
ANXIETY AND DEPRESSION IN FIBROMYALGIA PATIENTS A BLINDED CONTROLLED EVALUATION AS MEASURED BY STANDARDIZED PSYCHOMETRIC INTERVIEW SCALES.

NJ Krag J Norregaard, JK Larsen, B Danneskiold-Samsøe Department of Psychiatry and Department of Rheumatology, Frederiksberg Hospital, Copenhagen, Denmark.

Objective: To evaluate the presence of psychopathology in fibromyalgia patients, compared to a control group of patients with rheumatoid arthritis or lumbar disc herniation.

Methods: 49 fibromyalgia patients, 25 rheumatoid arthritis patients and 8 patients with lumbar disc herniation, all with daily pain, were interviewed blinded, using standardized psychometric scales. Pain was scored on a visual analogue scale. Results: On Bech Rafaelsen's melancholy scale fibromyalgia patients scored $12(9-16)$ as compared to $6(2,5-9)$ in the control group $(p<0,001)$. On the atypical depression scale fibromyalgia patients scored $12(8,5-14,5)$ as compared to $5(2,5-8,5)$ in the control group $(p<0,001)$. On the Hamilton anxiety scale fibromyalgia patients scored $19(13,5-23)$ as compared to $8(4-13)$ in the control group $(p<0,001)$. On the Newcastle scale, all patients in both groups scored below the limit for endogenous depression. There was a correlation between pain and psychometric scoring in both groups. The fibromyalgia group scored higher on the pain scale than the control group did. After correcting for differences in pain score, the fibromyalgia patients still scored higher than the control group on Bech Rafaelsen's melancholy scale, on the atypical depression scale and on the Hamilton anxiety scale. Conclusions: The fibromyalgia patients appear to be more anxious and depressed than other patients with chronic pain. Our data support the view, that the depression in fibromyalgia patients is a nonendogenous type of depression.
PSYCHOPATHOLOGICAL PARAMETERS IN SURGICAL PATIENTS G.N. Christodoulou, A. Voulgari, M. Papanikolaou, L. Lykouras, B. Alevizos Athens University Department of Psychiatry, 74 Vas. Sophias Aven., 11528 Athens, Greece.

Perioperative anxiety and depression in 162 surgical patients of good physical status aged between 18 and 60 years were studied.

Zung's Anxiety and Depression scales, Langner's Questionnaire for Psychopathology, Eysenck's Personality Questionnaire (E.P.Q.), SchallingSifneos' Alexithymia Scale, Foulds'Hostility Questionnaire and the Social Readjustment Rating Scale by Holmes and Rahe were used. Demographic, psychological and clinical parameters were also investigated. Anxiety and depression of the patients both pre- and post-operatively were associated with high neuroticism, high psychoticism, low extroversion, more psychotraumatic events during the last year, more fears concerning anaesthesia and surgery and increased postoperative pain. In conclusion, psychopathology influences the psychological and physical condition of the patients in the perioperative period.
PSYCHOLOGICAL EVOLUTION AND ASSESSMENT AFTER HEART TRANSPLANTATION

J.M.Triffaux ${ }^{*}$ J. Bertrand ${ }^{\star}$, O. Bertrand, J.C. Demoulin, J.O. Defraigne, H. Kulbertus.

"Hôpital de jour "La Clê", CHU Sart-Tilman (Liège, Belgium)

Background: Cardiac transplantation is a major stress factor and psychiatric evaluation is now routinely proposed in order 1) to detect possible contraindications and 2) to offer psychological support to the patient and his family.

Methods: Different tests are used to study: General Health (GHQ), Anxiety (STAI), Depression (BDI), Perceived Social Support (PSSS), Personal Reaction Inventory (PRI), Personality (MMPI). Alexithymic patients have difficulty identifying and describing their feelings, their cognitive style is concrete and reality-based, they have impoverished inner emotional and fantasy lives. We have applied the TAS-20 items scale questionnaire to evaluate alexithymia in a group of transplanted patients and a control group with no psychiatric or somatic event.

Results: From January 1990 to December 1992, thitty patients were asked to fill the questionnaire before and after the transplantation. There were 26 males and 4 females. Mean age was $57+1-7$ Years (extremes: 41-67). Control group $(n=31,27$ males and 4 females) mean age $56+l-5$ years There was no significant difference in transplantated patients before and after transplantation. TAS-20 score in transplantated patients was $54+1-13$ (28-76) versus $45+/-(24-72)$ in the control group $(p=0,009)$.

Conclusions: 1) Alexithymic profile seems to be more frequent in heart tansplanted patients. 2) As alexithymic patients are prone to psychosomatic diseases, alexythymic evaluation should be part of the psychological evaluation in heart transplantation candidates. 3) if alexithymia may be of prognostic significance in heart transplantation deserves further consideration. 